\section{PMO-147 RECOMBINANT ERYTHROPOETIN SUPPORT IS EQUALLY EFFECTIVE IN THE TREATMENT OF HCV PRE AND POST TRANSPLANT}

doi:10.1136/gutjnl-2012-302514b.147

A Barnabas, ${ }^{*}$ D Joshi, K Agarwal. Institute for Liver Studies, Kings College Hospital, London, UK

Introduction Recombinant EPO is often used in an attempt to improve Hepatitis $\mathrm{C}$ virus (HCV) treatment outcomes in complex patient groups. We assessed if its use was as effective in the posttransplant population as in a non-transplant group that also required EPO support. There is currently an absence of clear guidelines in this area and little evidence to support its efficacy overall.

Methods Between December 2009 and July 2010, 57 patients were prescribed EPO while undergoing HCV treatment. In this group, 13 patients who had undergone liver transplantation were identified. These were matched by HCV, genotype, gender and stage of liver fibrosis with a non-transplant group also treated with EPO. Numbers of patients being re-treated for HCV were similar in transplant and non-transplant groups (6/13 and $7 / 15$ respectively). Results 3/15 (19\%) in the non-transplant group and 1/13 $(7.7 \%)$ patients in the transplanted group achieved a sustained virological response to treatment. Discontinuation rates, however, were lower in the non-transplant group (4 vs 8 , OR 10.4). The initial $\mathrm{Hb}$ was not significantly different between the two groups (mean 12.78 in the transplant group and 13.42 in the non-transplant group, $\mathrm{p}=0.39$ ). Mean decrement in $\mathrm{Hb}$ was similar in transplant and non-transplant groups over the first 8 weeks of treatment (3.07 vs $2.54 \mathrm{~g} / \mathrm{dl})$ and the mean time to nadir in haemoglobin was similar in both groups (16 vs 19 weeks). 7/15 (47\%) (non-transplant vs 7/13 (54\%) transplant patients had a $\mathrm{Hb}$ decrement $>4 \mathrm{mg} / \mathrm{dl}$ on treatment (OR 0.83). Five patients (38\%) in the transplant group and 7 $(47 \%)$ in the non-transplant group required EPO prior to treatment week 8. Median treatment duration was shorter in the transplant group (24 vs 48 weeks). Ribavirin dosages varied widely in both groups and there was significant variation in ribavirin dose reduction strategies. One patient in the non-transplant group developed a deep vein thrombosis and one patient in the transplant group had a CVA while on treatment with EPO.

Conclusion Anaemia related to hepatitis $C$ treatment both presents and is managed similarly in both transplant and non-transplant difficult-to-treat populations. The risk of thrombotic events with EPO use is significant should be carefully borne in mind when initiating EPO support.

Competing interests None declared

\section{PM0-148 EVALUATION OF LIVER FIBROSIS IN YOUNG ADULT PATIENTS WITH CHRONIC HEPATITIS B VIRUS}

doi:10.1136/gutjnl-2012-302514b.148

${ }^{1} \mathrm{C}$ Patel, ${ }^{* 1} \mathrm{U}$ S Gill, ${ }^{1} \mathrm{~W}$ Alazawi, ${ }^{2} \mathrm{P}$ Shaw, ${ }^{3} \mathrm{E}$ Alstead, ${ }^{4} \mathrm{Y}$ Kallis, ${ }^{4} \mathrm{P}$ Kooner, ${ }^{4} \mathrm{R}$ Marley, ${ }^{5} \mathrm{~S}$ Naik, ${ }^{6} \mathrm{~J}$ ChinAleong, ${ }^{7} \mathrm{R} D$ Goldin, ${ }^{1} \mathrm{G}$ R Foster, ${ }^{1} \mathrm{P}$ T F Kennedy. ${ }^{1}$ Department of Hepatology, Blizard Institute of Cell \& Molecular Science, Barts and The London School of Medicine \& Dentistry; ${ }^{2}$ Department of Pharmacy, Whipps Cross University Hospital NHS Trust, London, UK; ${ }^{3}$ Department of Hepatology, Whipps Cross University Hospital NHS Trust, London, UK; ${ }^{4}$ Department of Hepatology, Barts and The London NHS Trust, London, UK; ${ }^{5}$ Department of Paediatric Gastroenterology \& Hepatology, Barts and The London NHS Trust, London, UK; ${ }^{6}$ Department of Histopathology, Barts and The London NHS Trust, London, UK; ${ }^{7}$ Liver \& GI Pathology, Imperial College, London, UK

Introduction Current guidelines do not recommend treatment of Chronic Hepatitis B (CHB) in young adults as they are believed to be immune tolerant with minimal liver damage. Recently we demonstrated that young $\mathrm{CHB}$ patients have evidence of immune activity comparable to older patients. Therefore, a proportion of young patients will develop fibrosis as a consequence of immune mediated liver injury. Despite this, the most appropriate modality to assess liver fibrosis remains controversial. We compare the utility of liver biopsy and digital image analysis with FibroScan for assessment of $\mathrm{CHB}$ in young adults.

Methods Young adult CHB patients were followed in a dedicated clinic and seen 3-monthly. Liver biopsy was performed where indicated and compared with FibroScan scores. Digital image analysis was performed on a selected number of Sirius red stained sections to evaluate percentage fibrosis. 167 patients were assessed; 101/167 (male $=63$ ); median age 26 (range 16-30) underwent liver biopsy and were included for analysis.

Results 47/101 patients were eAg+. Overall median ALT was 41IU/ L (range 14-426) \& HBV DNA was $5.22 \mathrm{log} \mathrm{IU} / \mathrm{ml}$ (range 1.4-9.3). Median Ishak fibrosis score was 1 (range 0-6); median FibroScan score was $5.95 \mathrm{kPa}$ (range 2.6-35.2). There was no difference in the FibroScan score in patients with advanced (F5/6) and minimal fibrosis (F0/1); median FibroScan scores were 6 and 5.95 respectively, $(p=n s)$. The correlation co-efficient between fibrosis and FibroScan was not statistically significant $(p=0.46)$. FibroScan scores were confounded by ALT; using Prati criteria to define normal ALT (female $<19$ IU/l, male $<30$ IU/1), we noted an ALT twice ULN revealed a higher FibroScan score, regardless of fibrosis $(p=0.05$ and 0.04 ; males and females respectively). On the contrary, digital image analysis more accurately reflected fibrosis stage irrespective of ALT.

Conclusion A proportion of young adult patients with $\mathrm{CHB}$ have significant liver disease on biopsy. This study highlights the limitations of FibroScan in assessing liver fibrosis in this cohort. Our data demonstrates the utility of digital image analysis in benchmarking fibrosis in young patients, potentially providing a more accurate methodology to assess progression of fibrosis over time.

Competing interests None declared.

\section{PMO-149 TENOFOVIR USE IN HEPATITIS B INFECTION IN PREGNANCY}

doi:10.1136/gutjnl-2012-302514b.149

G Chakrabarty, ${ }^{*}$ S Clark, D Forton. Department of Hepatology, St Georges University of London, London, UK

Introduction Antenatal screening and immunoprophylaxis reduces the perinatal transmission of hepatitis $B$ virus but vertical infection may occur with high level viraemia. Antiviral therapy during the $3 \mathrm{rd}$ trimester of pregnancy may reduce the incidence of intrauterine infection. Lamivudine treatment reduces vertical transmission if given with immunoglobulin and vaccination in mothers with viral loads $>10^{8} \mathrm{IU} / \mathrm{ml}$. There are no published trials for tenofovir in this context, although its use is supported by safety data from Antiretroviral Pregnancy Registryin USA (category B) with the added benefit of low viral resistance. Breastfeeding is not recommended as tenofovir is secreted in milk. Discontinuation of tenofovir after delivery carries the risk of hepatitis flares and liver decompensation. There are no guidelines for the use of antivirals in HBV-+ve pregnant women and treatment decisions are made on an individual basis.

Methods We initiated tenofovir $245 \mathrm{mg}$ in five HBV monoinfected women in the 3rd trimester to prevent vertical transmission. Demographic, virological and outcome data were collected.

Results The patients were newly diagnosed during antenatal screening. They were all $\mathrm{HBeAg}+\mathrm{ve}$ with high viral loads and normal ALT, presumed immunotolerant. All women wished to breastfeed and four were advised to stop tenofovir at delivery but 